\title{
Validation of a Flexible Multibody Belt-Drive Model
}

\author{
Gregor Čepon ${ }^{1}$ - Lionel Manin² - Miha Boltežar ${ }^{1, *}$ \\ 1 University of Ljubljana, Faculty of Mechanical Engineering, Slovenia \\ ${ }^{2}$ Université de Lyon, CNRS, INSA de Lyon, France
}

In the past decade the applicability of belt-drives has been extended significantly due to their increased reliability. With automotive engines it is now common to join a large number of belt-drives into a single, long belt-drive with several tensioner pulleys. However, these belt-drives can exhibit complex dynamic behaviors; therefore it is very important to predict the dynamics response of such systems using validated numerical models.

The aim of this paper is to perform the validation of a developed belt-drive model. The validation of this belt-drive model was performed using a two-pulley belt-drive. The numerically obtained results are compared with experimental data under various operational conditions. Finally, the applicability of the belt-drive model is presented by simulating a serpentine belt-drive, considering non-steady, belt-drive operational conditions.

(C2011 Journal of Mechanical Engineering. All rights reserved.

Keywords: belt-drive, validation, ANCF, angular speed loss, serpentine belt-drive

\section{INTRODUCTION}

Belt drives are commonly used to transmit power in many engineering applications, such as automotive engines, industrial machines, etc. V-ribbed, belt-drive systems have become increasingly important to the automotive industry since their introduction in the late 1970s. Because of their simple installation and low maintenance, together with an ability to absorb shocks, they are frequently used instead of chain or geared transmission systems. However, they can exhibit complex dynamic behaviors, such as the transverse vibrations of the belt spans, tension fluctuations, sliding of the belt over the pulley, etc. It is therefore very important to predict the dynamic response of such systems using validated numerical models.

To ensure stable working conditions the dynamic response of such systems has been studied extensively. A review of the literature [1] identifies two well-defined groups of studies. The first group deals with the transverse belt span response [2] to [5] and the rotational response of the pulleys in the belt-drive [6] to [9]. The second group deals with describing the belt-pulley contact formulation [10] and [11]. As these two groups suffer an unsatisfactory connection to each other, Leamy and Wasfy [12] and [13] bridged this gap by developing a general, dynamic finite-element model that includes frictional contact. Most recently, Čepon and Boltežar [14] presented an improved belt-drive model using the absolute nodal coordinate formulation (ANCF). The belt pulley contact forces were formulated as a linear complementarity problem (LCP), which enabled the incorporation of the discontinuous Coulomb friction law.

For any reliable simulations of belt-drives along with a validated numerical model, the proper material and contact parameters should be obtained. In [15], Čepon et al. presented methods for identifying the stiffness and damping for V-ribbed belts. Experimental studies of the contact between a grooved pulley and a V or V-ribbed belt are presented in [11] and [16]. In [16] the authors identified the contact parameters that are suitable for incorporation into the planar, multibody, belt-drive model that is presented in [14]. The procedure includes an experimental measurement of the contact-penalty parameters as well as a measurement of the friction coefficient.

The aim of this paper is to validate the belt-drive model developed by Čepon and Boltežar [14]. This validation involved using the two-pulley belt-drive. The numerically obtained results were compared with the experimental data under various operating conditions. The belt-drive

*Corr. Author's Address: University of Ljubljana, Faculty of Mechanical Engineering, 
material and the contact parameters used in the numerical model were obtained from [15] and [16]. Finally, the applicability of the belt-drive mode is presented by simulating the dynamic response of the complex, serpentine belt-drive with tensioners, which are common in automotive engines.

This paper is organized as follows. In Section 1 the belt-drive model is briefly presented. The validation procedure of the belt-drive model is presented in Section 2. In Section 3 the dynamic simulation of the serpentine belt-drives is presented. Finally, the conclusions are drawn in Section 4.

\section{NUMERICAL BELT-DRIVE MODEL}

The numerical belt-drive model is based on multibody system dynamics together with the absolute nodal coordinate system (ANCF) [14]. An ANCF is proposed that can be used in large rotation and deformation analyses of flexible bodies that undergo arbitrary displacements. The belt is modeled as a collection of two-dimensional beam elements that are based on the element originally proposed by Berzeri and Shabana [17]. The authors in [15] additionally supplemented the above-mentioned beam element with a damping mechanism. The system of equations of motion, including all the beam elements and the constraint equations describing the connectivity constraints, can be written as:

$$
\left[\begin{array}{cc}
\mathbf{M}_{\mathrm{B}} & \mathbf{C}_{e \mathrm{~B}}^{T} \\
\mathbf{C}_{e \mathrm{~B}} & \mathbf{0}
\end{array}\right]\left\{\begin{array}{c}
\ddot{\mathbf{e}} \\
\lambda_{\mathrm{B}}
\end{array}\right\}=\left\{\begin{array}{c}
\mathbf{Q}_{f}+\mathbf{Q}_{e \mathrm{~B}} \\
\mathbf{Q}_{d \mathrm{~B}}
\end{array}\right\},
$$

where $\mathbf{M}_{\mathrm{B}}$ is the constant mass matrix of the belt, $\mathbf{C}_{e \mathrm{~B}}$ is the Jacobian of the constraint equations and $\lambda_{B}$ is the vector of Lagrange multipliers. The vector $\mathbf{Q}_{e \mathrm{~B}}$ is the generalized force vector that includes external forces, $Q_{f}$ is the generalized force vector due to the stiffness and damping forces, and $\ddot{\mathbf{e}}$ includes the accelerations of generalized coordinates of all the belt elements.

Each belt element has five possible contact points, which are equally spaced along the length of the element, Fig. 1.

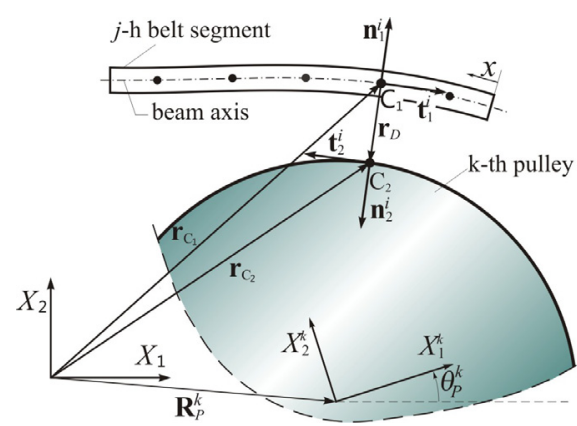

Fig. 1. Belt pulley contact formulation

In the tangential direction the discontinuous Coulomb friction law is used. In order to compute the possible sticking forces the contact problem in the tangential direction has to be formulated as a linear complementarity problem [14]. By using the discontinuous Coulomb friction law and linear complementarity problem it is possible to identify sticking and sliding contact. Moreover, events such as the transition from sticking to sliding or sliding to sticking are also possible. The equations of motion, including the contact forces between the belt and the pulley, can be written as:

$$
\ddot{\mathbf{q}}_{r}=\mathbf{H}_{F}\left(\mathbf{W}_{N} \lambda_{N}+\mathbf{W}_{T} \lambda_{T}\right)+\mathbf{h},
$$

where $\lambda_{N}$ and $\lambda_{T}$ are the contact forces in the normal and tangential directions. Variable $\mathbf{q}_{\mathbf{r}}$ includes accelerations of generalized coordinates, $\mathbf{H}_{F}$ matrix presents the system mass properties, $\mathbf{W}_{N}$ is a kinematic matrix associated with normal contacts, $\mathbf{W}_{T}$ is a kinematic matrix associated with tangential contacts and $\mathbf{h}$ is the vector of external forces [14]. Finally, the contact problem can be formulated in the form of a linear complementarity problem. As reported in [14], this formulation leads to an accurate prediction of the belt-pulley contact forces, even when non-steady, belt-drive operational conditions are considered. For a detailed description of the belt-drive model and the contact formulation between the belt and the pulley the interested reader is referred to [14] and [15].

\section{VALIDATION OF THE BELT-DRIVE MODEL}

\subsection{Two-Pulley Belt-Drive Experimental Setup}

The validation of the belt-drive model was made using a two-pulley belt-drive, as shown in 
Fig. 2. A V-ribbed belt with five ribs and a K-rib section (5PK) was used.

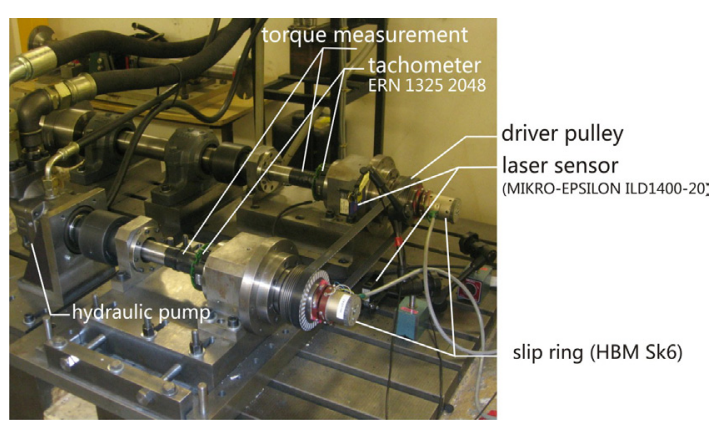

Fig. 2. Two-pulley belt drive experimental setup

The material properties and contact parameters of the belt are given in [15] and [16] and presented in Table 1. The measured friction characteristics [16] are given in Fig. 3. The friction coefficient depends on the normal contact force and the sliding velocity in the contact.

Table 1.Two-pulley belt-drive parameters

\begin{tabular}{|l|c|c|}
\hline \multicolumn{1}{|c|}{ Parameter } & Sym. & Value \\
\hline $\begin{array}{l}\text { Driver and driven } \\
\text { pulley }\end{array}$ & $R$ & $0.05 \mathrm{~m}$ \\
\hline Belt length & $L_{\mathrm{J}}$ & $1.2 \mathrm{~m}$ \\
\hline $\begin{array}{l}\text { Mass moment of } \\
\text { inertia of the pulley }\end{array}$ & $J_{\mathrm{T}}$ & $0.013 \mathrm{kgm}^{2}$ \\
\hline $\begin{array}{l}\text { Pulley central } \\
\text { distance }\end{array}$ & $L_{\mathrm{c}}$ & $0.45 \mathrm{~m}$ \\
\hline $\begin{array}{l}\text { Density of the belt } \\
\text { material }\end{array}$ & $\rho$ & $0.096 \mathrm{~kg} / \mathrm{m}$ \\
\hline Axial stiffness & $E A$ & $30400 \mathrm{~N} / \mathrm{rib}$ \\
\hline $\begin{array}{l}\text { Viscoelastic damping } \\
\text { factor }\end{array}$ & $c A$ & $4.1 \mathrm{Ns} / \mathrm{rib}$ \\
\hline Bending stiffness & $E I$ & $5.2 \cdot 10^{-3} \mathrm{Nm}^{2} / \mathrm{rib}$ \\
\hline Rayleigh parameter & $\alpha$ & $2.8 \mathrm{~s}^{-1}$ \\
\hline Rayleigh parameter & $\beta$ & $1.8 \cdot 10^{-3} \mathrm{~s}$ \\
\hline
\end{tabular}

In order to verify the numerical model the numerical results were compared with the experimentally obtained results. The driver pulley was rotated by DC motor and the torque on the driven pulley was achieved by a piston hydraulic pump. The angular velocity of driven and driver pulley was measured with HAIDENHAIN ERN1325 2048 precise optical encoders. The

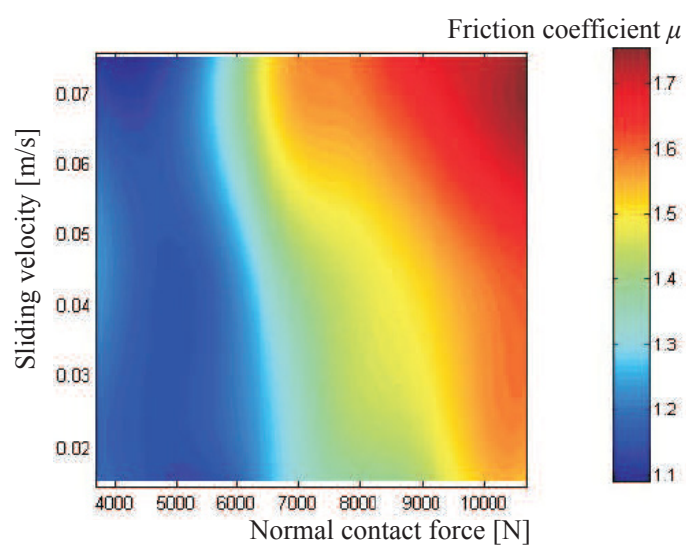

Fig. 3. Friction coefficient versus the normal contact force and the relative tangential velocity

initial tension of the belt spans was set by changing the mid-distance of the pulleys.

The torque on the driver and the driven shaft was measured with strain gages. Along with this measurement, the transverse displacements of the upper (tight) and lower (slack) spans were also measured using laser-displacement sensors. Based on the displacements we were able to deduce the natural frequencies of the belt spans for the given operational conditions.

\subsection{Comparison between the Simulated and Experimental Results}

The comparison between the numerically and experimentally obtained results was preformed with two different initial belt tensions and several angular velocities of the driver pulley.

The numerical two-pulley belt-drive model [14] is presented in Fig. 4 and the belt-drive parameters are given in Table 1.

Points $\mathrm{A}$ and $\mathrm{B}$ denote the measurement location of the transverse displacement of the upper and lower belt spans. The discretization of the belt involved using 48 beam elements. Three contact points were proposed in the contact between one beam element and the pulley.

As the beam elements have equal length, the contact points are equally spaced along the length of the belt. Thus, the periodic excitation due to the discretization is incorporated into the numerical model. This can be avoided by randomly changing the length of the beam elements, which also affects on the location of the contact points. 


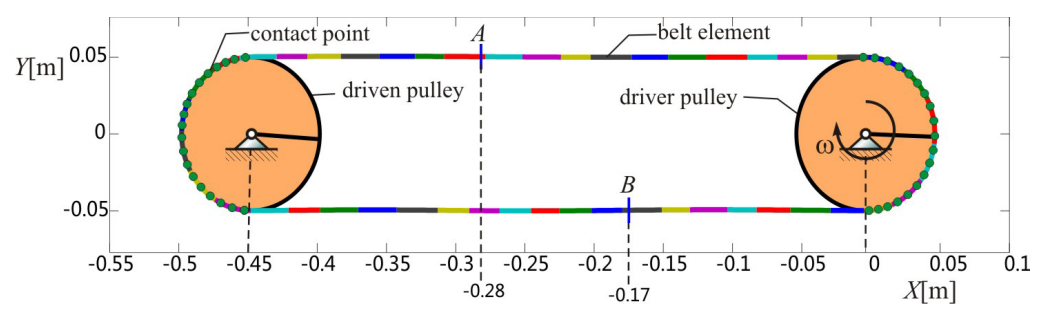

Fig. 4. Numerical belt-drive model

The random length of the beam elements was computed using the following equation:

$$
\begin{aligned}
& L_{\text {rand }}^{i}=\frac{L_{J}}{n_{e}}(1+0.12 \cdot \text { rand }), \\
& \sum_{i=1}^{n_{e}} L_{\text {rand }}^{i}-L_{J} \leq 1 \cdot 10^{-4} m,
\end{aligned}
$$

where $L_{J}$ is the length of the belt, $L_{\text {rand }}^{i}$ is the $i^{\text {th }}$ length of the beam segment and rand is the random function that generates random numbers in the interval $[-1,1]$.

\subsection{Angular Speed Loss}

The angular speed loss between the driver and the driven pulley has a significant effect on the efficiency of the power-transmission. Therefore, an accurate prediction of angular speed loss is of great importance, especially in the automotive industry. There are three contributions to consider when dealing with angular speed loss [11]:

- the creep along belt,

- the radial compliance,

- the shear deflection.

Creep represents the belt stretching in the slip contact region between the belt and the pulley. Radial compliance represents the radial deformation as the rubber layer is subjected to a radial load when it is pressed against the pulley (Fig. 5).

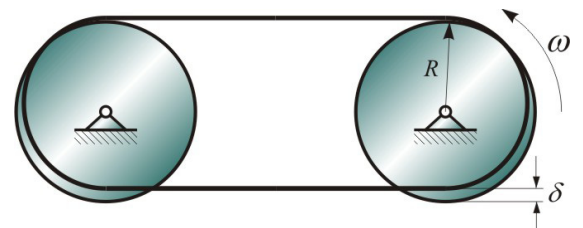

Fig. 5. Radial compliance
For this reason the radius at the entry and exit regions of the belt-pulley contact are not equal, which then affects the angular speed loss. The shear deflection along the belt is caused by the frictional forces that are transferred from the belt-pulley contact though the rubber to the cord layer (Fig. 6).

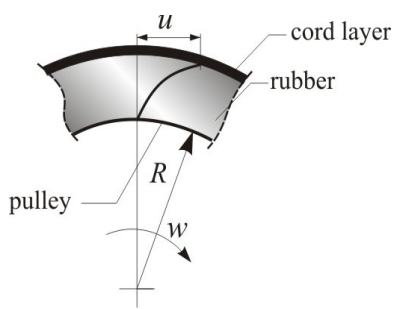

Fig. 6. Shear deflection of the rubber layer

Usually, the analytical equation that estimates the angular speed loss is given as [10]:

$$
\Delta \omega=\left(1-\frac{2 T_{0} R-M+2 R k}{2 T_{0} R+M+2 R k}\right) \omega_{1},
$$

where $T_{0}$ is the initial tension, $R$ is the radius of the pulley, $M$ is the torque on the pulley, $k$ is the stiffness of the belt and $\omega_{1}$ is the angular velocity of the driver pulley. Eq. (4) only accounts for the creep theory and neglects all the other phenomena that impact the angular speed loss. However, in our numerical model, along with creep, the radial compliance is also taken into account.

In Fig. 7 the computed angular speed losses with the analytical Eq. (4) and the developed numerical model are compared with the experimentally obtained angular speed losses. The measured speed losses are much higher than those predicted by the analytical model or our numerical model. The grey region in Fig. 7 presents the difference between the results obtained with the numerical model of the belt-drive and the experimentally obtained results. 
This difference is due to the tangential deformation of the belt-rubber layer, which is not accounted for in the numerical belt-drive model. It can be seen that the effect of the shear deformation becomes significant at higher torque values. The blue region presents the difference between the numerical model and the analytical model. This difference is the effect of the radial compliance, which is not accounted for in the analytical solution. In our numerical model this radial deformation is taken into account through the elastic component of the penalty contact force.

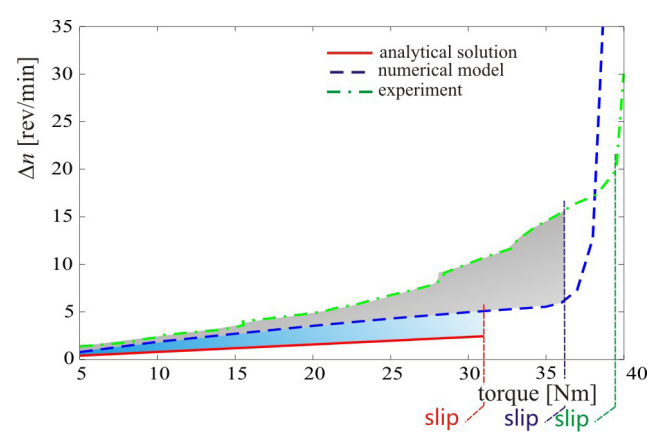

Fig. 7. Angular speed loss between the driver and the driven pulley $\left(\omega=62.8 \mathrm{rad} / \mathrm{s}, T_{0}=310 \mathrm{~N}\right)$

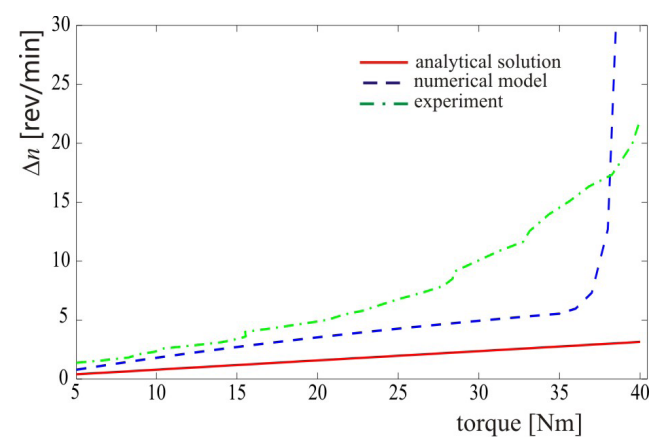

Fig. 8. Angular speed loss between the driver and the driven pulley $\left(\omega=22.6 \mathrm{rad} / \mathrm{s}, T_{0}=310 \mathrm{~N}\right)$

From the comparisons presented in Fig. 7 and Fig. 8 it is evident that the analytical model gives the poorest prediction of the angular speed loss. Moreover, the point of belt slippage is not correctly determined. However, our numerical model gives more reliable predictions of the angular speed loss, especially at low torques. In addition, the numerical model can quite accurately predict the point of the belt slippage. This accurate prediction is the result of a well-identified friction coefficient and the rigidity of the belt bending [16] and [17]. At high values of torque, which occur near the slippage point, the axial force in the slack span results mainly due to the bending of the belt span. The belt bending stiffness forces the belt to bulk outwards, which generates additional axial forces in the belt. This acts as a tensioner, which supplies the minimal axial force in the slack span.

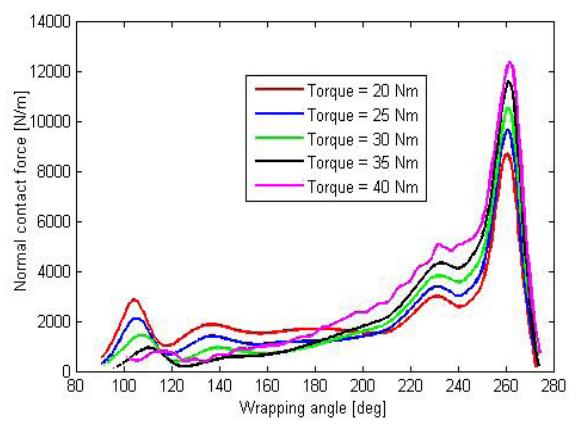

Fig. 9. Belt normal contact force versus wrapping angle

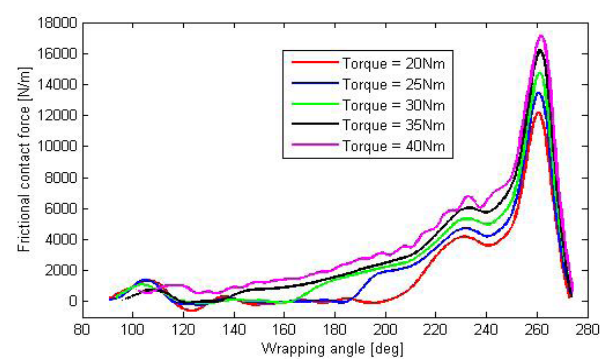

Fig. 10. Belt tangential contact force versus wrapping angle

The normal and frictional forces between the belt and the driven pulley obtained using the numerical model are presented in Figs. 9 and 10. It can be seen that the numerical model predicts the peaks of the normal and tangential forces at the entry and exit section of the belt, which was also reported in [14].

\subsection{Belt Span Transverse Response}

The transverse belt-span response was measured with two laser sensors positioned at the upper and lower belt spans. The experimentally obtained results were compared with the result obtained using the numerical belt-drive model. 
The excitation of the belt span is caused by the roughness of the belt surface, the radial clearance, of the bearing, the wear of the belt grooves, etc.

a)

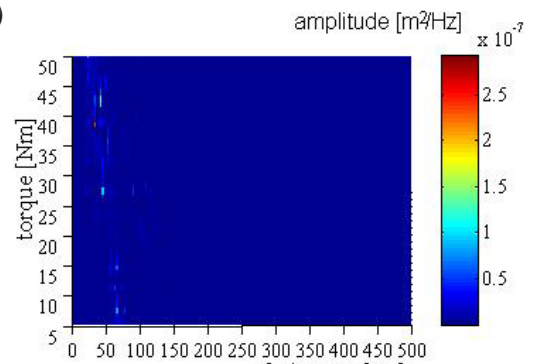
frekvency $[\mathrm{Hz}]$

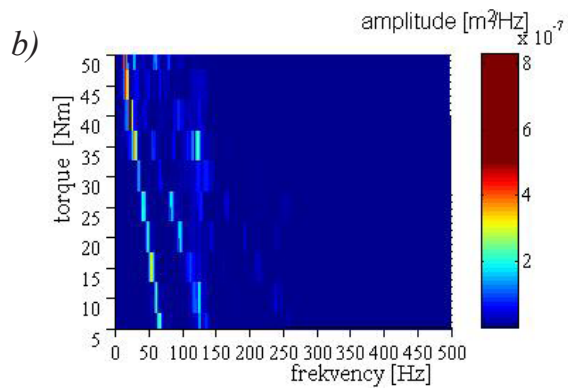

Fig. 11. Magnitude spectra of the slack belt span response at point $A$ versus the torque on the driven pulley; a) experiment, b) numerical simulation

a)

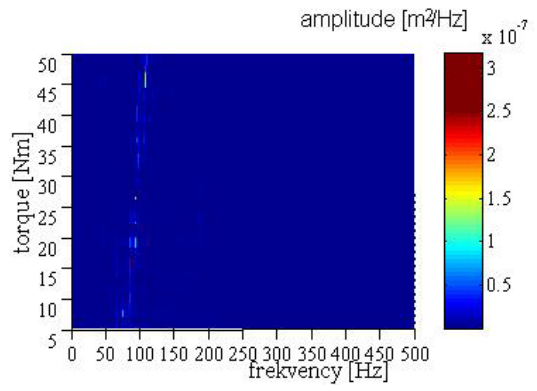

b)

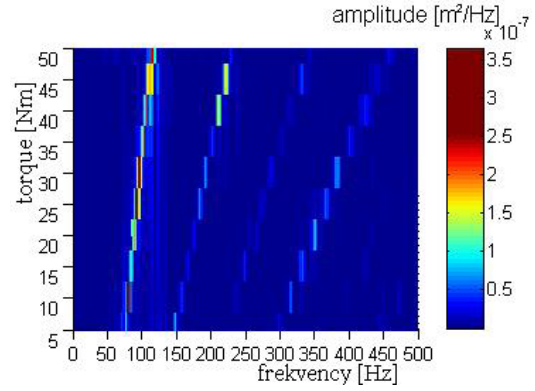

Fig. 12. Magnitude spectra of the tight belt span response at point $B$ versus the torque on the driven pulley; a) experiment, b) numerical simulation
Due to the random nature of the excitation it is practically impossible to model all these phenomena in a numerical belt-drive model.

Thus, the time histories obtained from the experiment and the numerical model cannot be directly compared. However, we can compare the frequency contents of both signals.

In Figs. 11 and 12 the magnitude spectra of the tight and slack and belt spans versus the torque on the driven pulley are presented. The belt-span responses are obtained at a driver pulley angular velocity $\omega=62.8 \mathrm{rad} / \mathrm{s}$ and initial tension of the belt $T_{0}=411 \mathrm{~N}$. From the magnitude spectra the dependence of the belt-span natural frequencies on the drive-pulley torque can be obtained. This torque correlates directly with the value of the axial force in the slack and tight spans. The increase in the torque, when slack span is considered, causes a decrease in the belt's axial force. This can be seen from Fig. 11a, where the dependence of the first natural frequency versus the torque can be identified. From Fig. 11b, which presents the results of the numerical simulation, even higher natural frequencies can be identified. As in the case of the experimentally obtained natural frequencies, the first natural frequency is the most pronounced. Moreover, the agreement between the experimentally identified natural frequencies and the frequency identified from the simulations is good. Similar conclusions can be drawn when a tight belt span is considered in Fig. 12. Here, the increase in the torque on the driven pulley causes an increase of the axial force of the belt. Thus, by increasing the torque the natural frequencies are increased. Also in this case, good agreement between the experiment and the numerical simulation was observed.

\section{SERPENTINE BELT-DRIVE MODELING}

In this section the applicability of the presented belt-drive model will be presented for modeling serpentine belt-drives with tensioners, which are common in automotive engines. The simulation will be performed for a serpentine beltdrive with three pulleys and two tensioners, Fig. 13.

Non-steady, belt-drive operational conditions are considered with the following angular velocity profile: 
$\omega=\left\{\begin{array}{cc}60 \cdot \frac{t}{0.3} & 0 \mathrm{~s} \leq t<0.3 \mathrm{~s} \\ 60+10 \sin (2 \pi 40(t-0.3)) & t \geq 0.3 \mathrm{~s}\end{array}\right.$.

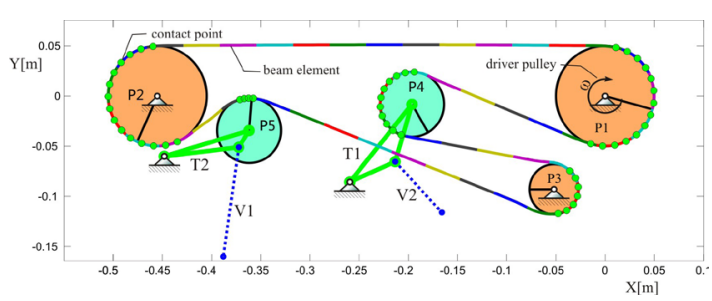

Fig. 13. Serpentine belt-drive

The torque on the driven pulley is assumed to be constant and is equal to $M=45 \mathrm{Nm}$. The variables V1 and V2 denote the system of the spring and dashpot, which are connected in parallel.

In Fig. 14 the numerically obtained axial force in the tight (between pulleys P1-P2) and slack belt spans (between pulleys P3-P5) is presented. Due to the non-steady, belt-drive operational conditions, the axial forces in the belt are time dependent. The variation of the belt's axial force is considerably smaller in the slack belt span, which is due to the use of tensioners.
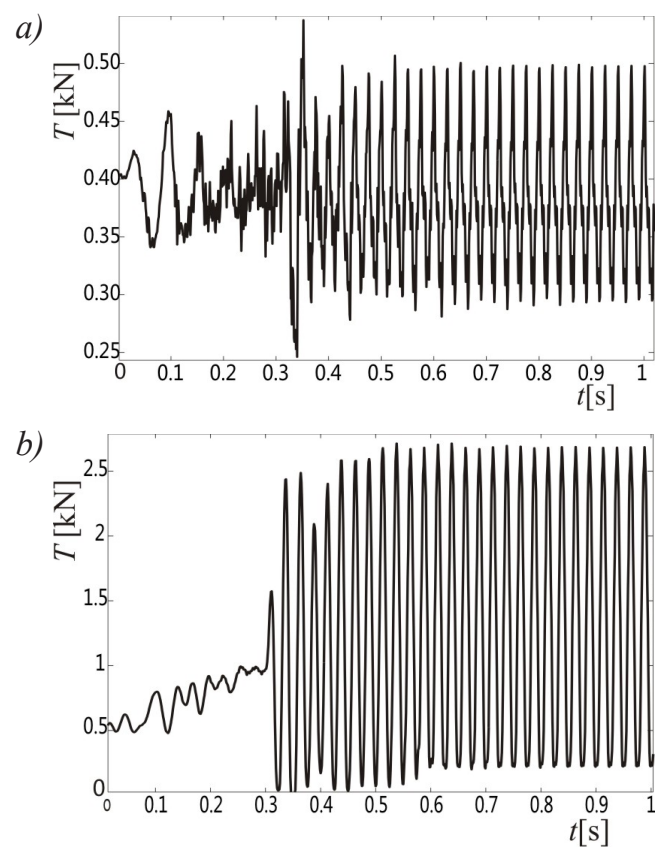

Fig. 14. Force in the belt span; a) force in the belt span between pulleys P3-P5, b) force in the belt span between pulleys P1-P2
In the process of the numerical simulation it is also possible to deduce the rotation of the tensioner arm, as shown in Fig. 15.

The angular velocities of the pulley in the belt-drive are presented in Fig. 16. It is evident that the angular-speed loss between the driver (P1) and the pulley (P2) is practically negligible. This is achieved with two tensioners, which supply sufficient axial tension and consequently friction, even at high torques.

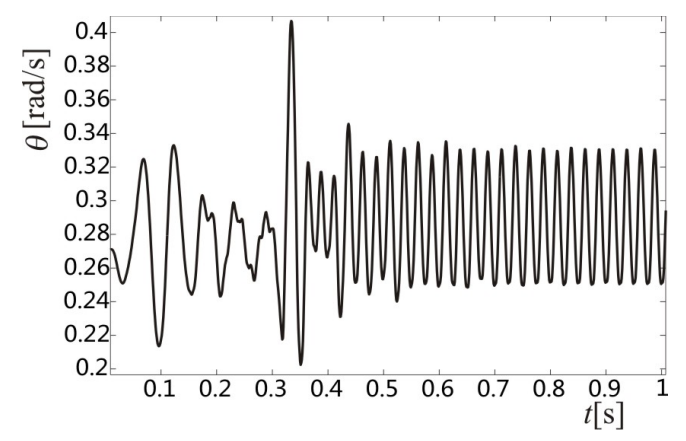

Fig. 15. Rotation of tensioner arm T2

A dynamic simulation of belt-drives enables us to model the dynamic response of a complex serpentine belt. In this way, the belt-drive can be optimized from the view of the operational and shape parameters.

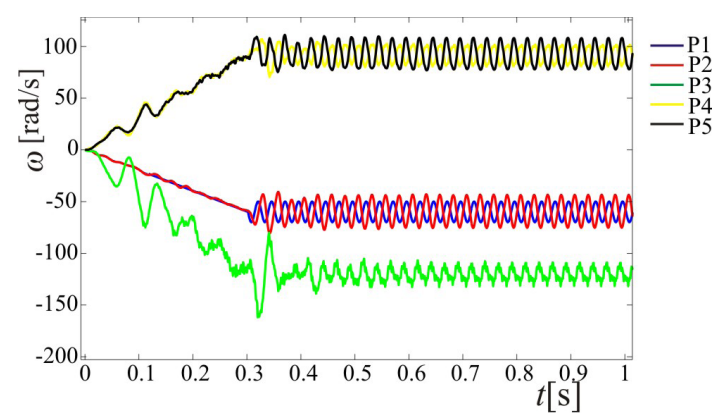

Fig. 16. Pulley angular velocities

\section{CONCLUSION}

In this paper a belt-drive model using the absolute nodal coordinate formulation is presented and a validation study is performed. A two-pulley experimental setup was proposed. Good agreement between the experimental and numerically obtained results was found. It was also shown that the numerical belt-drive model gives 
a reliable prediction of the angular-speed loss, especially at low torques. Moreover, it accurately predicts the point of belt slippage. Considering the belt-span vibrations, the agreement between the experimentally identified natural frequencies and the frequency obtained from the simulation is good.

Finally, the applicability of the belt-drive model was presented by simulating a complex belt-drive with two tensioners.

In further work the parametric and sensitivity analysis should be performed in order to identify influential parameters. Moreover, the belt model should be improved by using higher order elements. This would lead to a reduction of element number and degrees of freedom and consequently, to computationally more efficient numerical algorithm.

\section{REFERENCES}

[1] Abrate, S. (1992). Vibrations of belts and belt-drives. Mechanism and Machine Theory, vol. 27, p. 645-659.

[2] Pramila, A., Laukanen, J., Pautamo, M. (1983). Vibration of axially moving material using the FEM. The ASME, paper no. 83DET-96.

[3] Pellicano, F., Catellani, G., Fregolent, A. (2004). Parametric instability of belts: theory and experiments. Computers and Structures, vol. 82 p. 81-91.

[4] Čepon, G., Boltežar, M. (2007). Computing the dynamic response of an axially moving continuum. Journal of Sound and Vibration, vol. 300, p. 316-329.

[5] Qie, G., Dukkipati, R., Zhu, J., Qatu, M. (2008). Vibrations and instability of frontend accessory drive belt system. International Journal of Vehicle Noise and Vibration, vol. 4, p. 247-268.

[6] Hwang, S.J., Perkins, N.C., Ulsoy, A.G., Meckstroth, R.J. (1994). Rotational response and slip prediction of serpentine belt-drive systems. ASME Journal of Vibration and Acoustics, vol. 116, p. 71-78.

[7] Kraver, T.C., Fan, G.W., Shah, J.J. (1996). Complex modal analysis of a flat belt pulley system with belt damping and coulomb- damped tensioner. ASME Journal of Mechanical Design, vol. 118, p. 306-311.

[8] Iwatsubo, T., Hasegawa, K., Arii, S., Shiohata, K. (1997). The formulation and dynamic analysis of a multiple belt system. Journal of Sound and Vibration, vol. 205, p. 293-307.

[9] Manin, L., Play, D., Soleilhac, P. (2000). Experimental validation of a dynamic numerical model for timing belt-drives behaviour simulation. ASME DETC, Baltimore.

[10] Bechtel, S.E., Vohra, S., Jacob, K.I., Carlson, C.D. (2000). The stretching and slipping of belts and fibers on pulleys. Journal of Applied Mechanics, vol. 67, p. 197-206.

[11] Gerbert, G. (1996). Belt slip - a unified approach. Journal of Mechanical Design, vol. 118 , p. 432-438.

[12] Leamy, M.J., Wasfy, T.M. (2002). Transient and steady-state dynamic finite element modeling of belt-drives. Journal of Dynamic Systems, Measurement, and Control, vol. 124, p. $575-581$.

[13] Leamy, M.J., Wasfy, T.M. (2005). Timeaccurate finite element modelling of the transient, steady state and frequency responses of serpentine and timing beltdrives. International Journal of Vehicle design, vol. 39, p. 272-297.

[14] Čepon, G., Boltežar, M. (2009). Dynamics of a belt-drive system using a linear complementarity problem for the belt-pulley contact description. Journal of Sound and Vibration, vol. 319, p. 1019-1035.

[15]Čepon, G., Manin, L., Boltežar, M. (2009). Introduction of damping into the flexible multibody belt-drive model: A numerical and experimental investigation. Journal of Sound and Vibration, vol. 324, 283-296.

[16] Čepon, G., Manin, L., Boltežar, M. (2010). Experimental identification of the contact parameters between a V-ribbed belt and pulley. Mechanism and Machine Theory, vol. 45, p. 1424-1433.

[17]Berzeri, M., Shabana, A.A. (2000). Development of simple models for the elastic forces in the absolute nodal co-ordinate formulation. Journal of Sound and Vibration, vol. 235, p. 539-565. 